Relations industrielles

Industrial Relations

\title{
Labor Economics and Labor Relations, by Lloyd G. Reynolds, Prentice-Hall, Englewood Cliffs, 1970, 5e ed., 692 pp.
}

\section{Jean-Pierre Beaulieu}

Volume 26, numéro 1, 1971

URI : https://id.erudit.org/iderudit/028207ar

DOI : https://doi.org/10.7202/028207ar

Aller au sommaire du numéro

Éditeur(s)

Département des relations industrielles de l'Université Laval

ISSN

0034-379X (imprimé)

1703-8138 (numérique)

Découvrir la revue

Citer ce compte rendu

Beaulieu, J.-P. (1971). Compte rendu de [Labor Economics and Labor Relations, by Lloyd G. Reynolds, Prentice-Hall, Englewood Cliffs, 1970, 5e ed., 692 pp.] Relations industrielles / Industrial Relations, 26(1), 254-255.

https://doi.org/10.7202/028207ar

Tous droits réservés (C) Département des relations industrielles de l'Université Laval, 1971
Ce document est protégé par la loi sur le droit d'auteur. L'utilisation des services d'Érudit (y compris la reproduction) est assujettie à sa politique d'utilisation que vous pouvez consulter en ligne.

https://apropos.erudit.org/fr/usagers/politique-dutilisation/ 
caines et britanniques, mais qui portent sur des sujets d'intérêt canadien. Les annotations accompagnant chaque thèse reproduisent, d'une façon générale, la table des matières de ces thèses.

L'ouvrage est composé de trois parties, la première traitant des thèses poursuivies dans les universités canadiennes, la deuxième traitant de celles poursuivies dans les universités américaines et la troisième faisant état de celles effectuées en Grande-Bretagne. Suivent, en appendice, la classification de l'American Economic Association et une liste choisie des sources consultées.

Des tableaux présentés en préface, indiquent la distribution des thèses recensées selon le pays et l'époque où elles ont été poursuivies, et selon le diplôme auquel elles ont conduit leurs auteurs (Tableau 1). Ils indiquent encore comment ces thèses se répartissent en fonction du sujet traité et du pays en cause (Tableau 2), ainsi qu'en raison, en ce qui a trait au Canada, des universités qui ont octroyé les diplômes.

Il est intéressant de constater, par exemple, que, même si le nombre de thèses de doctorat a progressé avec les années, au Canada, les thèses de maîtrise sont plus de dix fois supérieures en nombre, alors que celles provenant des Etats-Unis et de Grande-Bretagne comprennent presque deux fois plus de thèses de doctorat que de thèses de maîtrise. Il y a là matière à réflexion pour le monde universitaire canadien.

Autre fait intéressant à noter, le nombre de thèses de maîtrise, émanant des universités Laval et de Montréal et portant sur le «travail » l'emporte par une très forte marge sur celles consacrées aux autres sujets compris dans ce recueil et qui émanent de ces mêmes universités. On peut sans nul doute attribuer ce fait à l'existence, dans ces deux universités, de départements de relations industrielles depuis longtemps actifs dans la formation professionnelle de spécialistes en ce domaine.

S'ajoutant aux autres publications vouées aux mêmes fins, le répertoire de Wood, Kelly et Keimar constitue un outil précieux pour les chercheurs en sciences sociales.

\section{Jean-Réal CARDIN}

Labor Economics and Labor Relations, by Lloyd G. Reynolds, Prentice-Hall, Englewood Cliffs, 1970, 5e ed., 692 pp.

Labor Economics and Labor Relations de Lloyd G. Reynolds, un classique qui en est à sa cinquième édition, la première datant de 1949 . Ce volume constitue presqu'une somme des théories contemporaines en économique du travail et en relations du travail pourrait porter lieu à une très longue analyse. Notre propos n'en est pas là. Nous commentons plutôt les éléments nouveaux ou modifiés de cette dernière édition par rapport à la précédente.

Le volume est constitué de trois grandes parties: Economics of the Labor Market; Collective Bargaining : Institution and Procedure; Collective Bargaining: Economic Impact. Les sujets ou chapitres à l'intérieur de chacune d'elles sont ceux qu'on retrouve dans des ouvrages du même genre, tels ceux de Cartter, Bloom \& Northrup, Kuhn et d'autres 1 .

Dans la présente édition de son volume, Reynolds a inversé la place de l'analyse économique et l'a mise au début. Deux points sont à l'appui de ce changement: l'économique du travail englobe tout individu de la maind'oeuvre, syndiqué ou non. Le marché du travail dans lequel cet individu se situe a le même cadre général modifié ou non par les négociations collectives. Il est donc bon d'analyser d'abord ce cadre économique avant de s'attaquer aux modifications qu'y apportent les syndicats. L'importance croissante de l'analyse économique et des techniques de recherche qui s'y rattachent constituent le second point.

Un autre argument est également apporté pour justifier cette nouvelle présentation et il a particulièrement retenu notre attention :

"The policy issues examined in Part
I seems likely to be of greatest in-
terest to students during the $1970 \mathrm{~s}$.
They included such things as : the
meaning of equal eductional oppor-
tunity and feasible ways of attaining

1 Cartter, A.M., Theory of Wages and Employment; Bloom, C.F. et Northrup, H.R., Economics of Labor Relations; Kuhn, A., Labor: Institutions and Economics; Woods, H.D., Ostry, S., Labour Policy and' Labour Economics in Canada. 
it ; the reason for the disadvantaged economic position of black workers and the main lines of remedial action; the problem of structural unemployment and its reduction through retraining and relocation programs; the problem of restraining wage and price increases during periods of high employment; the dimensions of poverty and new antipoverty proposals such as the negative income tax. These and other issues of labor market policy are discussed more fully than in previous editions".

Ces sujets méritent d'être signalés et constituent, à notre avis, l'intérêt de cette nouvelle édition d'un volume déjà considéré comme un classique, ce qui revient presque à dire qui a fait sa marque demeure source de référence mais n'est plus à la fine pointe des discussions d'aujourd'hui. Par l'étude de tels sujets Reynolds anime l'économique du travail, la situe sur un autre plan que le plan abstrait de la pure analyse théorique, il la place dans le contexte social d'aujourd'hui et commente la marge qu'il y a entre le modèle et le réel.

C'est une optique semblable qui marquait le volume de Woods et Ostry dans certains chapitres. Nous sommes heureux de constater que peu à peu des économistes, auteurs, tentent de combler le fossé entre la théorie et le réel. Galbraith aurait-il été compris?

\section{Jean-Pierre BEAULIEU}

Psychologie du groupe, par M.A. Robert, manuel théorique et pratique de l'animateur. Bruxelles, Coll. «Humanisme d'aujourd'hui », Editions Vie Ouvrière, 1969, 173 pp.

Voici un petit ouvrage extrêmement bien fait dans sa conception, sa présentation et sa formulation. Il comporte une partie théorique sur les groupes ainsi qu'une partie consacrée aux techniques de groupe. L'auteur est un véritable pédagogue; il sait amener le lecteur à comprendre des notions difficiles qu'il explique avec une clarté et une précision sans verser dans des simplifications superficielles. Avec l'importance que prend aujourd'hui le travail en groupe, cet ouvrage est destiné à rendre des services inappréciables à tous ceux qui étudient les phénomènes de groupe et leur réalité concrète.

Gérard DION
Les Business Schools Américaines, par Michel Chevalier et Jean François de Chorivit, Paris, Entreprise moderne d'édition, 1970, 122 pp.

Petit volume intéressant sur les écoles américaines d'administration écrit par les auteurs à partir de leur expérience comme étudiants à Harvard. C'est un peu une autre découverte de l'Amérique à un niveau micro-économique ; découverte qui entraîne la comparaison avec le système français dont on démontre les grandes faiblesses et l'anachronisme face aux besoins de la société actuelle. Le système américain d'éducation, surtout au niveau gradué, est très bien développé. Les ressources financières et humaines ne manquent pas au point où les institutions de haut-savoir se permettent une saine concurrence dans la qualité de leur enseignement, dans la compétence de leurs diplômés. Si cette situation a fait réfléchir les auteurs français, elle pourrait peut-être porter à la réflexion certaines personnes du milieu universitaire québécois et c'est pourquoi nous leur recommandons cette lecture.

\section{Jean-Pierre BEAULIEU}

L'entretien d'appréciation : six dialogues entre chef et subordonné, par N.R. Maier, Paris, Entreprise Moderne d'Edition, 1968, 290 pp.

L'ouvrage de Norman Maier est déjà connu aux Etats-Unis et au Canada puisqu'il a été publié en langue anglaise en 1958. Comme il a connu une très grande diffusion, l'Entreprise moderne d'édition en a fait une excellente traduction.

On sait que l'entretien d'appréciation cause énormément d'embarras à tout cadre dirigeant qui doit à des moments précis rencontrer ses subordonnés pour leur communiquer ce qu'il pense d'eux et surtout de leur comportement au travail. Même s'ils appréhendent cette confrontation, la plupart des gens dans une situation de subalternes désirent être appréciés à leur 《juste valeur» et connaître ce que leur supérieur pense d'eux. C'est là une responsabilité auquelle peut difficilement se soustraire quelqu'un qui coordonne et supervise le travail d'autres personnes. 